\title{
Cloud Microphysics Impact on Hurricane Track as Revealed in Idealized Experiments
}

\author{
ROBERT G. FOVELL AND KRISTEN L. CORBOSIERO \\ Department of Atmospheric and Oceanic Sciences, University of California, Los Angeles, Los Angeles, California \\ HUNG-CHI KUO \\ Department of Atmospheric Science, National Taiwan University, Taipei, Taiwan
}

(Manuscript received 2 July 2008, in final form 23 December 2008)

\begin{abstract}
Analyses of tropical cyclones created in an idealized environment reveal how and why cloud microphysical assumptions can influence storm motion, including speed and direction. It is well known that in the absence of a mean flow, a leading factor in storm propagation is the establishment of "beta gyres" owing to planetary vorticity advection by the storm's circulation. Previous research demonstrated that tangential winds well beyond the core influence storm motion by helping to determine the gyres' orientation and intensity.

Microphysical assumptions, especially involving average particle fall speeds, can strongly influence the winds at outer radius. More specifically, microphysics modulates the radial distribution of column-average virtual temperature, which largely determines the radial surface pressure gradient and therefore the winds because they tend to be in gradient balance beyond the core. Microphysics schemes can differ markedly with respect to average fall speed, depending on the complexity of the scheme and how interactions among condensation types are handled. Average fall speed controls the outward movement of particles produced in the eyewall into the anvil, where they can influence the environment through cloud-radiative interactions and phase changes.

With the assistance of some special sensitivity tests, the influence of microphysics and fall speed on radial temperature gradients, leading to different outer wind strengths and tracks, is shown. Among other things, this work demonstrates that the treatment of outer rainbands in operational models can potentially influence how simulated storms move, thus affecting position forecasts.
\end{abstract}

\section{Introduction}

Clouds have a tremendous impact on weather on many scales. They produce heating and cooling, transport mass vertically and horizontally, and disturb their surroundings, generating precipitation, intense winds, and turbulence. Clouds can also have more subtle effects, for instance, through anvils that can shade the surface, thereby influencing horizontal temperature gradients (e.g., Markowski et al. 1998), or by acting as ducts for gravity waves (e.g., Fovell et al. 2006). When the clouds, their constituent particles, and/or their effects are unresolvable by a numerical model, parameterizations become necessary.

Corresponding author address: Robert G. Fovell, UCLA Atmospheric and Oceanic Sciences, 405 Hilgard Ave., Los Angeles, CA 90095-1565.

E-mail: rfovell@ucla.edu
Weather forecasting models at operational resolutions (horizontal grid spacing $>10 \mathrm{~km}$ at this writing) are required to compensate for unresolvable convective activity with convective and cloud microphysics parameterizations (cf. Stensrud 2007). Convective schemes, such as Kain-Fritsch (Kain and Fritsch 1993) and Betts-Miller (Betts and Miller 1986), attempt to specify those gridscale effects of clouds that cannot be resolved. Cloud microphysics refers to the processes that govern the growth, fallout, and evolution of condensation particles associated with grid-scale condensation. Both parameterizations participate in representing the convective activity without double counting its thermodynamic impacts (Zhang et al. 1988).

Fovell and Su (2007, hereafter FS07) showed that varying cloud microphysics assumptions, together with cumulus parameterizations, can have a material effect on hurricane tracks simulated in a regional-scale model at $30-\mathrm{km}$ horizontal resolution, even in relatively short-range 
(2 day) forecasts. This was demonstrated in a physicsbased ensemble forecasting experiment for Hurricane Rita (2005), which came ashore near the Texas-Louisiana border. For simulations commencing $54 \mathrm{~h}$ prior to landfall, FS07's ensemble predicted landfall locations ranging between Lake Charles, Louisiana, to Rockport, Texas, which is a span of over $200 \mathrm{~km}$. The contemporaneous National Hurricane Center (NHC) consensus revealed a nearly identical ensemble spread from the bulk of its dynamical model members (after excluding a few obvious outliers).

With 30-km grid spacing, inclusion of both parameterizations was necessary to produce model tropical cyclones having intensities and other characteristics comparable to observations. However, FS07 also conducted a higher-resolution $(12 \mathrm{~km})$ experiment that permitted realistic storms with only microphysics active in the case of Rita. A substantial spread of tracks and forecast landfall locations was still obtained. In that and in an idealized experiment, it was also apparent that microphysical assumptions directly or indirectly modulated storm structure, which FS07 noted could be one reason why the storms tracked differently.

While numerous authors report microphysical influences on hurricane intensity (e.g., Lord et al. 1984; Wang 2002; McFarquhar et al. 2006; Zhu and Zhang 2006), few have been concerned with, or have acknowledged, track sensitivity. This paper is concerned with how and why cloud microphysics assumptions can influence hurricane motion. It makes use of simulations that are made in a dynamical framework as similar as possible to that employed in operational forecasting, but sufficiently simplified so that the microphysical impacts can be identified and assessed.

The format of this paper is as follows: The model design is introduced in section 2 . The influence of microphysics on storm track is analyzed in section 3. Section 4 presents sensitivity tests, revealing even more ways microphysical assumptions can affect motion. A summary is contained in section 5 .

\section{Model}

In their idealized experiments, FS07 employed a modified "real data" version of the Weather Research and Forecasting (WRF) model (inspired by Hill and Lackmann 2009) that retained earth curvature but had no land, a uniform sea surface temperature (SST) of $29^{\circ} \mathrm{C}$, and a background environment based on Jordan's (1958) Caribbean hurricane season composite sounding, although without any initial winds whatsoever. A coherent vortex was bred through incorporation of a synoptic-scale warm, moist anomaly into the initial con- dition and then integrated for a spinup period $\tau_{s}$ during which the Kain-Fritsch convective parameterization (CP) was used and cloud microphysics was inactive. After $\tau_{s}$, the CP scheme was turned off and one of three microphysics parameterizations (MPs) was activated in all three telescoping domains, the outer being a 3240-km square with $27-\mathrm{km}$ resolution and the innermost being $669 \mathrm{~km}$ on a side with $3-\mathrm{km}$ grid spacing. The outer domain was intended to capture the entire environmental response to the hurricane. In the vertical, 30 levels were used, with highest resolution concentrated near the surface.

The present study makes use of a slightly modified experiment that largely reproduces FS07's results. We again consider the Kessler and Lin-Farley-Orville (LFO) five-class and the WRF three-class single-moment (WSM3) MPs as being representative of the variety of single-moment parameterizations available. Kessler is a warm rain scheme possessing only two forms of condensation: free-floating cloud droplets and swiftly falling rain. LFO adds to this three forms of ice: free-floating crystals, slowly falling low-density aggregates (snow), and graupel particles having more moderate densities and fall speeds. The WSM3 is a simple ice scheme in which condensation above the freezing level is treated as either ice crystals or snow.

In contrast with FS07, the present experiment employed a more recent version of WRF (2.2.1). Surface physics code modifications resulted in somewhat weaker storms; otherwise, only minor differences were noted. Most of the simulations employed a 50-mb model top and used the Rapid Radiative Transfer Model (RRTM) radiation parameterization (Mlawer et al. 1997). Among the sensitivity tests conducted (section 4) were runs with higher (10 mb) model tops and the Community Atmospheric Model (CAM) radiative scheme.

The analyses presented herein make use of vortexfollowing composites that are constructed for periods during which the storms exhibited structural and behavioral stability. The symmetric components reflect azimuthally averaged composite fields.

\section{Results}

Cyclone positions at 12 -h intervals commencing at $\tau_{s}+12 \mathrm{~h}$ for the three MP simulations are presented in Fig. 1. The results are broadly comparable to those in FS07 (their Fig. 4). As in that study, the tracks quickly diverged following the common startup, with the Kessler (K) storm swiftly propagating to the northwest whereas the two MPs considering ice produced slower, and ultimately more northward, motions. By $60 \mathrm{~h}$, even the LFO (L) and WSM3 (W) storms were separated by 


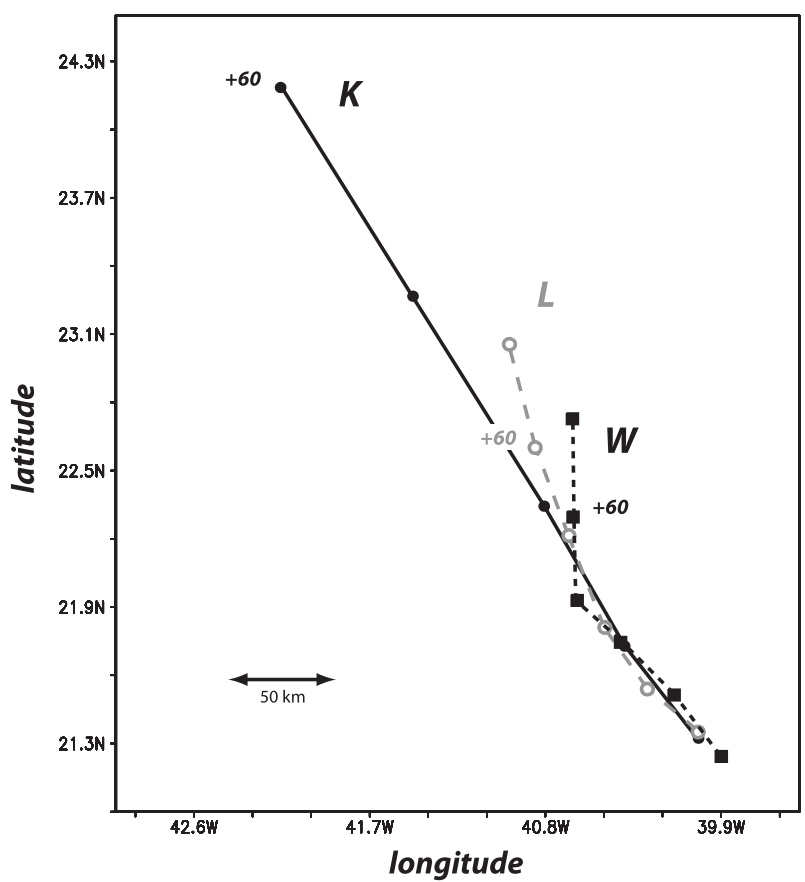

FIG. 1. Twelve-hourly positions (representing geometric centroids of sea level pressure) for the Kessler (K), LFO (L), and WSM3 (W) storms between $\tau_{s}+12 \mathrm{~h}$ and $\tau_{s}+72 \mathrm{~h}$. The Kessler position at $\tau_{s}+72 \mathrm{~h}$ is beyond the region depicted, which represents a portion of the innermost domain in which the horizontal grid spacing was $3 \mathrm{~km}$.

over $60 \mathrm{~km}$, and the Hurricane Rita (2005) landfall scenario demonstrates that a position discrepancy as ostensibly small as this can still have major consequences. Without imposed large-scale flow, these vortex motions represent self-propagation that has clearly been modulated by microphysics.

Information regarding storm intensity and size, including eye diameter, may be inferred from Fig. 2, which presents the radial variation of the $850-\mathrm{mb}$ symmetric wind speed component from the storm-following composites. This is intended to represent the flow at the top of the planetary boundary layer that is essentially uninfluenced by surface friction. As in FS07, the Kessler storm resulted in the widest eye and the strongest winds at larger radius, whereas for the two ice MP cases, the LFO storm was most intense and the WSM3 case had the most compact eye.

The tracks and storm structures in Figs. 1 and 2 bear a striking resemblance to that in Fig. 3 of Fiorino and Elsberry (1989, hereafter FE89). Using a nondivergent, barotropic model with no basic current, FE89 demonstrated that very different tracks and propagation speeds could be obtained by simply modifying the tangential winds in the outer part of the vortex. As in our experiment, the tracks included a rapid mover to-

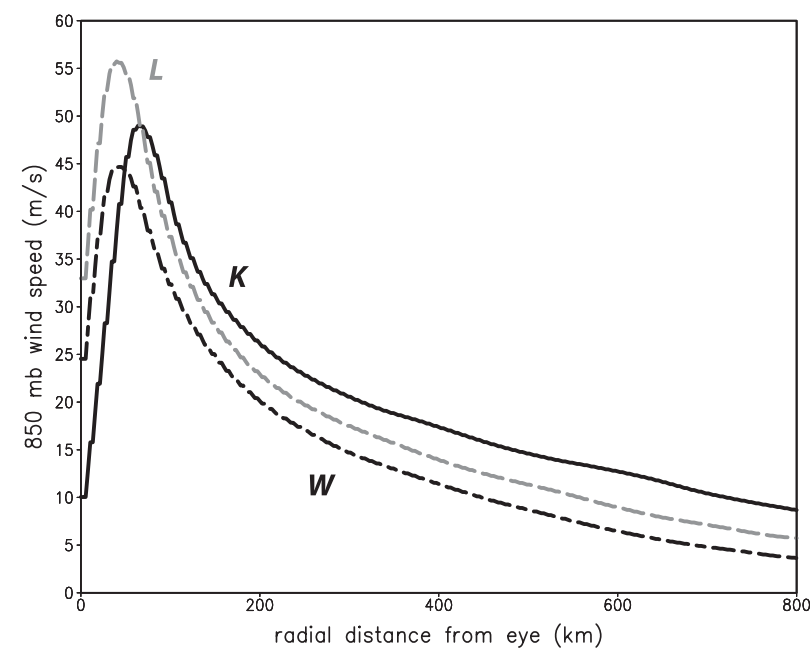

FIG. 2. Symmetric component of $850-\mathrm{mb}$ wind speed $\left(\mathrm{m} \mathrm{s}^{-1}\right)$, constructed from vortex-following composites over a 24-h period for the three MP storms. (K, L, and $\mathrm{W}$ are defined in Fig. 1.)

ward the northwest as well as slower, more northwarddirected storms. When FE89 varied only the winds closer to the eye (radius $r<300 \mathrm{~km}$ ), almost no difference in track or storm speed resulted (their Fig. 2).

FE89 explained storm motion in their model as resulting from a ventilation flow established across the vortex as a result of the establishment of beta gyres (Holland 1983; Chan and Williams 1987) that are a consequence of differential advection of planetary vorticity by the storm's cyclonic circulation. FE89 found that the winds at outer radius, being $300 \mathrm{~km}$ and farther from the eye in their study, strongly influenced storm motion because they determined the orientation and intensity of the gyres. Indeed, they also demonstrated that subtle variations in outer wind strength and radial variation could have a major impact on both storm track and speed.

In the present experiment, the simulated storms that had stronger outer winds propagated more quickly and along a more northwestward track. FE89 used a much simpler model than ours, and factors missing from their model can and will influence storm propagation and the flow across the vortex such as baroclinic processes (Wu and Emanuel 1993; Flatau et al. 1994; Wang and Holland 1996) and internal instabilities (Nolan et al. 2001; Marks et al. 2008). Nevertheless, the strength of the symmetric flow at large radius is considered here to be a leading explanatory term for hurricane motion in an environment with no initial basic current. Thus, an explanation will be given as to how and why microphysics has influenced the azimuthally averaged winds at large distances from the eye.

The first step is to interpret the radial variation of the winds in the context of surface pressure $\left(p_{s}\right)$ gradients, 


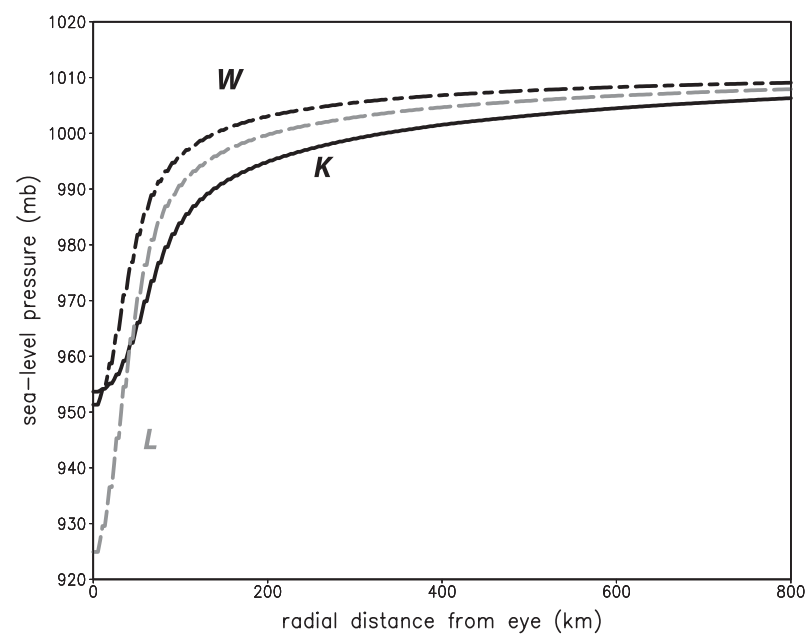

FIG. 3. As in Fig. 2, but for the symmetric component of sea level pressure.

which are illustrated in Fig. 3 for the three MP cases. The inviscid horizontal equation of motion in cylindrical coordinates applied at or near the surface is

$$
\frac{d u_{s}}{d t}-f v_{s}-\frac{v_{s}^{2}}{r}=-\frac{1}{\rho_{s}} \frac{\partial p_{s}}{\partial r}
$$

where $u_{s}$ and $v_{s}$ are the near-surface radial and tangential winds, respectively, $f$ is the Coriolis parameter, and $\rho_{s}$ is the sea level air density. If the flow is steady, which is a better assumption away from the vortex core, an approximation for $v_{s}$ is

$$
\bar{v}_{s} \approx \frac{-f r+\sqrt{(f r)^{2}+4 r \frac{1 \partial p_{s}}{\rho_{s} \partial r}}}{2} .
$$

The tangential winds approximated from (2) using Fig. 3's surface pressures are shown in Fig. 4, superposed on the radial wind profiles from Fig. 2. Beyond $r \approx 100 \mathrm{~km}$, the fit is excellent, which indicates that the symmetric component of the $850-\mathrm{mb}$ wind speed is well represented by the radial gradient of sea level pressure (SLP).

In the hydrostatic limit, the pressure beneath a column of depth $Z$ is related to the pressure at the top $\left(p_{Z}\right)$ and the column-average virtual temperature $\left(\bar{T}_{v}\right)$; that is,

$$
p_{s}=p_{Z} \exp \left(\frac{g Z}{R_{d} \bar{T}_{v}}\right),
$$

where $g$ and $R_{d}$ are the gravitational acceleration and dry air gas constant, respectively. The surface pressures estimated using $Z=14.5 \mathrm{~km}$ in (3) are superposed in Fig. 5 on the SLP profiles from Fig. 3. This fit is also excellent. Although it will be shown below that this height is within the anvil of at least some of the model

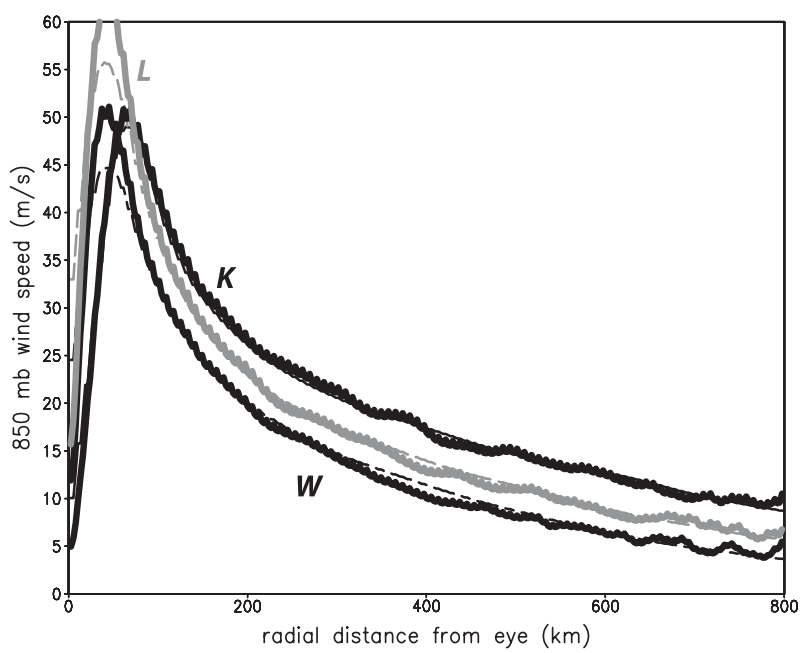

FIG. 4. As in Fig. 2, but with the approximation of the tangential wind $[(2)]$ superposed.

storms, it was chosen because the pressure of $141 \mathrm{mb}$ at this height varied little with radius or among the cases.

Therefore, by far the largest contributor to the surface pressure is the column average virtual temperature between the surface and the $14.5-\mathrm{km}$ level. The radial distribution of the symmetric component of $\bar{T}_{v}$ used in (3) is shown in Fig. 6. At larger radii, the virtual temperatures of the three MP storms vary by several degrees, with the Kessler storm having the highest temperatures. However, the winds reflect radial pressure gradients, which are hydrostatically related to temperature gradients, so the key discriminator among the storms is the larger radial variation of $\bar{T}_{v}$ beyond the core for the Kessler case.

The higher average virtual temperature and larger radial gradient in the Kessler simulation resulted in part from that storm having a more prominent upper tropospheric anvil than that produced by the two ice MPs. Vertical cross sections of total condensate, positioned west-east across the eye and averaged over a period of $12 \mathrm{~h}$, are shown in Fig. 7 for the three cases. The Kessler anvil is obviously thicker and wider and contains more mass than that produced by either ice scheme. The Kessler anvil has reached an enormous horizontal extent, with a diameter exceeding $2800 \mathrm{~km}$. Although somewhat discontinuous in nature and smaller in comparison to the Kessler storm anvil, the two ice MP schemes also generated substantial anvils that are wider than those documented in Kossin (2002) and comparable to the roughly $1300-\mathrm{km}$-wide $\left(13^{\circ}\right.$ of longitude at $27^{\circ} \mathrm{N}$ ) cloud shield of Hurricane Katrina (2005).

These anvil size differences may seem surprising at first. In squall lines, MPs with ice typically produce wider anvils (e.g., Fovell and Ogura 1988) because much 


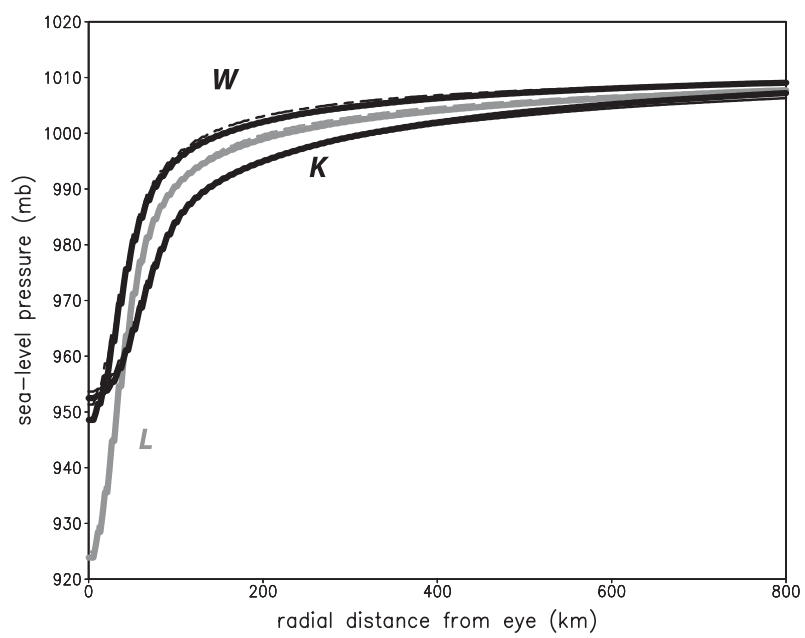

FIG. 5. As in Fig. 3, but with the estimated sea level pressure using (3) superposed.

of the condensate residing above the freezing level becomes ice crystals or low-density snow, both of which have smaller terminal velocities than raindrops. With a smaller average fall speed, the anvil can spread farther horizontally. The saturation vapor pressure difference between ice and liquid water is also relevant because condensation is more likely to form and persist when treated as solid water. In contrast, the Kessler scheme usually produces rain rather quickly, which leads to larger particle sizes and fall speeds throughout the cloud.

The only mechanism for producing new precipitation particles in the Kessler parameterization is the autoconversion process in which cloud droplets self-aggregate into drops having nonnegligible terminal velocities. Autoconversion usually depends on the presence of a critical concentration of cloud droplets in a given volume. Powerful updrafts in squall lines are prodigious generators of condensation and drive the (perhaps overly) rapid conversion of droplets to rain. In contrast, the characteristically weaker updrafts in hurricanes [only $5 \%$ exceed $5 \mathrm{~m} \mathrm{~s}^{-1}$, according to Black et al. (1996)] produce less condensation and so fewer new raindrops are produced, owing to the smaller droplet concentrations. The LFO and WSM3 schemes have several routes to produce precipitation and are thus more likely to create new precipitating particles above the freezing level in volumes lacking them. In these simulations, it was the Kessler case that had the smallest average particle fall speed outside the core and thus developed the most extensive anvil cloud.

Also shown in Fig. 7 are virtual temperature perturbations from the initial state. In all three simulations, the expected elevated temperatures in the eye region can be noted (e.g., Hawkins and Rubsam 1968), indicative of both storm intensity and eye diameter. For the

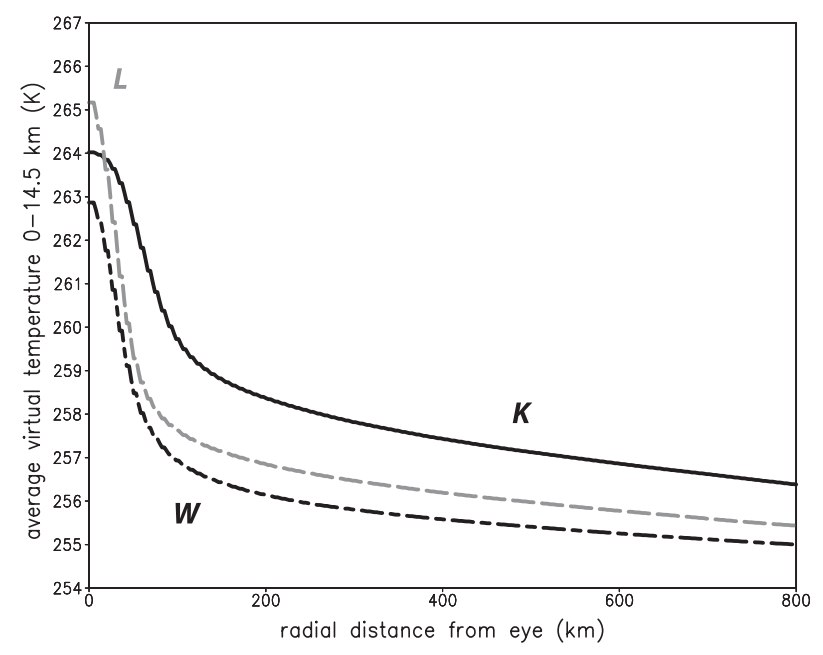

FIG. 6. As in Fig. 2, but showing the symmetric component of composite virtual temperature $(\mathrm{K})$ averaged between the surface and $14.5-\mathrm{km}$ levels.

two ice MP cases (Figs. 7b,c), positive perturbations extend radially outward from the core with maximum extents at the $9-10-\mathrm{km}$ level. Those perturbations are roughly comparable in scale and magnitude to those retrieved by the Advanced Microwave Sounding Unit sensor (Kidder et al. 2000; Knaff et al. 2004) and likely resulted from compensating subsidence in the region beyond the core. However, the Kessler storm had prominent warm regions beneath and cool regions above the horizontally extensive anvil. It will be shown in the next section that the extensive anvil in the warm rain storm impacted radiation calculations and resulted in modifications to the radial temperature and pressure gradients that ultimately influence the track.

Notice that the average virtual temperatures in Fig. 6 were computed at a level that excluded the cooling above the Kessler storm's anvil (see Fig. 7). Selection of a higher level decreases the column $\bar{T}_{v}$ for the warm rain case but also alters column top pressure $p_{Z}$ (not shown). In these simulations, the $14.5-\mathrm{km}$ level had the smallest pressure perturbations with respect to the initial state. Other levels increased the importance of $p_{Z}$ relative to $\bar{T}_{v}$ in (3), which complicated the interpretation of the hydrostatic effect of temperature on surface pressure and winds.

\section{Sensitivity tests}

The preceding results demonstrated that at least in idealized simulations having no initial flow, assumptions inherent in cloud microphysical schemes can have a first-order effect on the motion (speed and direction) of 

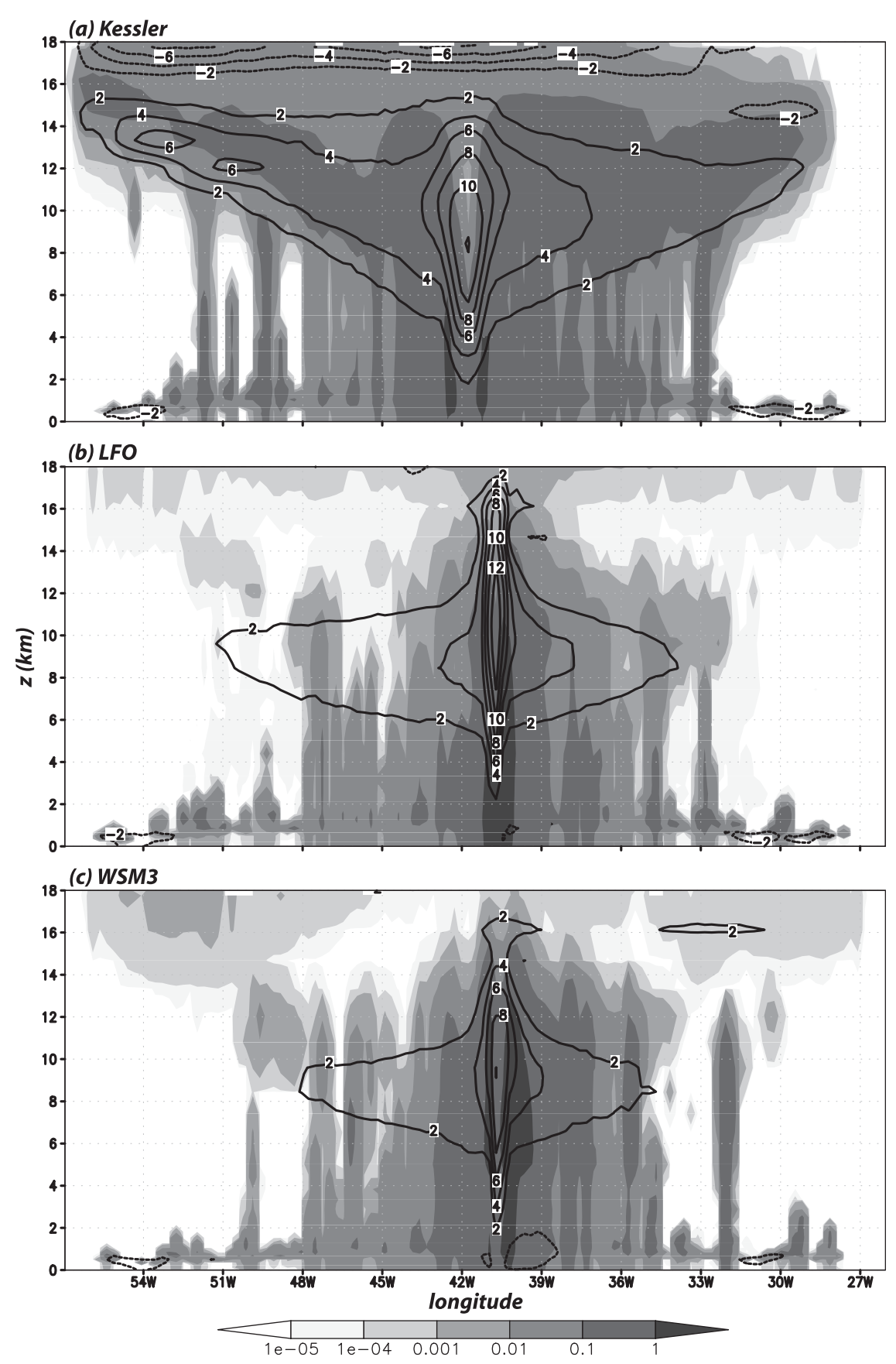

FIG. 7. Vertical cross sections of condensate $\left(\mathrm{g} \mathrm{kg}^{-1}\right.$; shaded according to scale at bottom) and virtual temperature perturbations from the initial state $(\mathrm{K}$; contoured at $2 \mathrm{~K})$ for the Kessler, LFO, and WSM3 storms, respectively, constructed from composite fields. Note that the shading scale exaggerates small values of condensation.

a tropical cyclone. In this section, the sensitivity to model depth, the radiation scheme, and particle fall speeds is investigated. Although the disparity between the two ice MP schemes was not insignificant, at least from an operational forecasting viewpoint, the purpose of this section is to elucidate the reasons why the Kessler results were so different. Note it is not necessary for the Kessler scheme to be a realistic microphysical parameterization for these results to be important or instructive. Once the dynamical linkage connecting microphysics to hurricane motion is established, more sophisticated MPs could be tweaked-in relatively subtle and even 


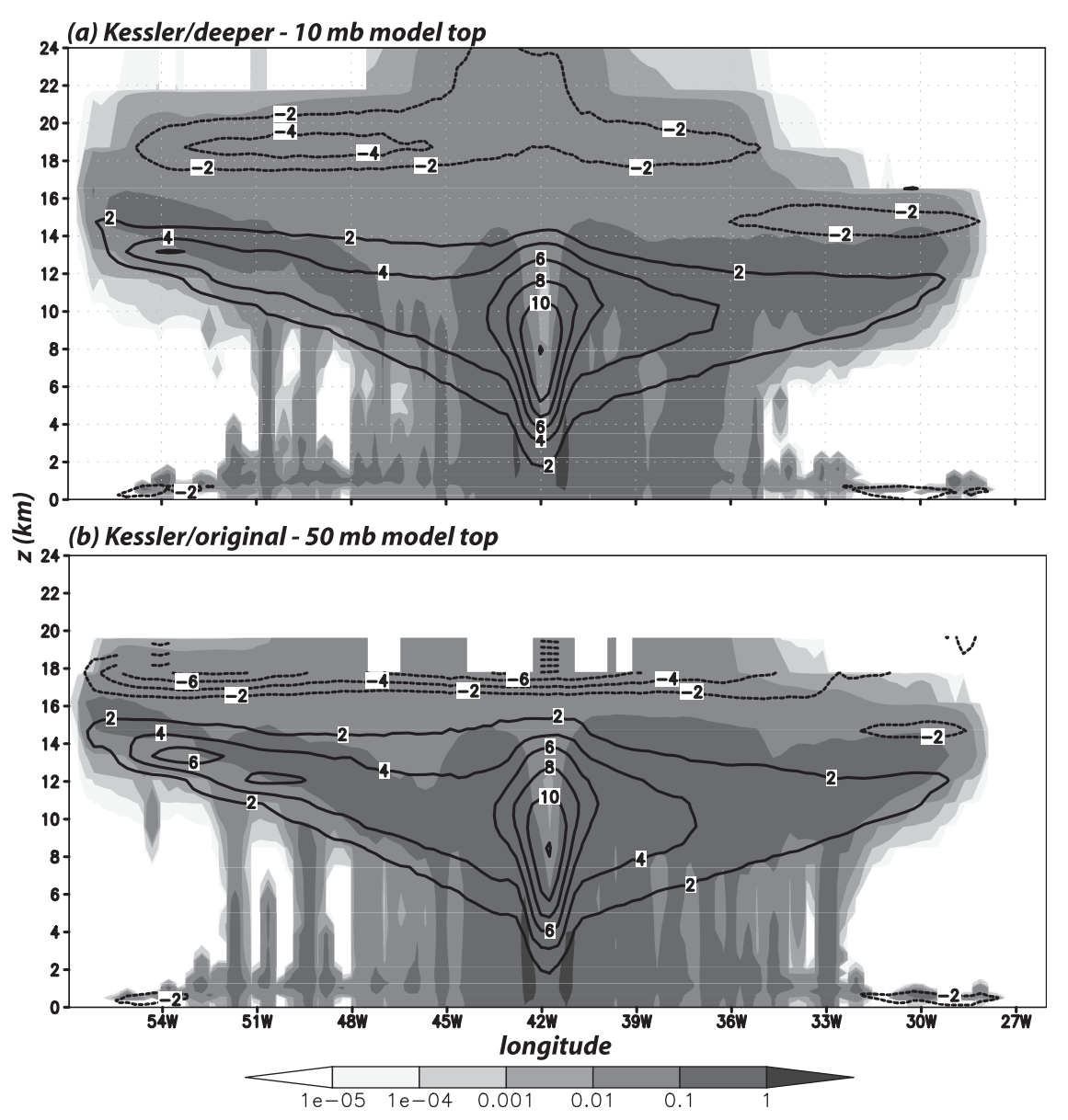

FIG. 8. As in Fig. 7, but for the deeper and original domain Kessler runs.

unobjectionable ways-to produce similar results. It is the dynamical linkages that are of interest.

\section{a. Domain depth and radiation experiments}

Increasing the domain depth to the $10-\mathrm{mb}$ level was not found to materially influence simulated storm structure or motion, independent of which MP was employed. Intercomparison of condensate and virtual temperature perturbation fields revealed no adverse impact arising from employment of a somewhat shallower domain. Figure 8 demonstrates this for the Kessler case, which was selected for presentation because the shallower version revealed temperature perturbations located relatively close to the original $(50 \mathrm{mb})$ model top (Fig. 7a). The deeper simulations employed the same number of vertical grid points, which resulted in a slightly smaller average vertical resolution.

Influence of the radiation scheme on the warm rain MP was also investigated (Figs. 9-11). Whereas the CAM radiation parameterization was found to produce results similar to those of the RRTM scheme, turning radiative processes off (simulation K/NORAD) had a major impact on storm structure and propagation, which resulted in a slower and more northward storm motion (Fig. 9) that closely resembles the storms simulated with the MP schemes having ice. In the K/NORAD simulation, the winds at larger radii were substantially weaker than when radiation was included (Fig. 10a). As expected from the reasoning in section 3, these differences in wind profiles may be traced to smaller radial temperature gradients beyond the core (Fig. 10b). Although the K/NORAD storm's upper tropospheric anvil remained extensive (Fig. 11), without the radiative effects little influence was exerted on the temperatures, the winds at larger radii, or the storm motion.

\section{b. Particle fall speed experiments}

Particle terminal velocity is likely the most significant factor in determining whether a MP will generate a substantial anvil cloud. However, such velocities depend 


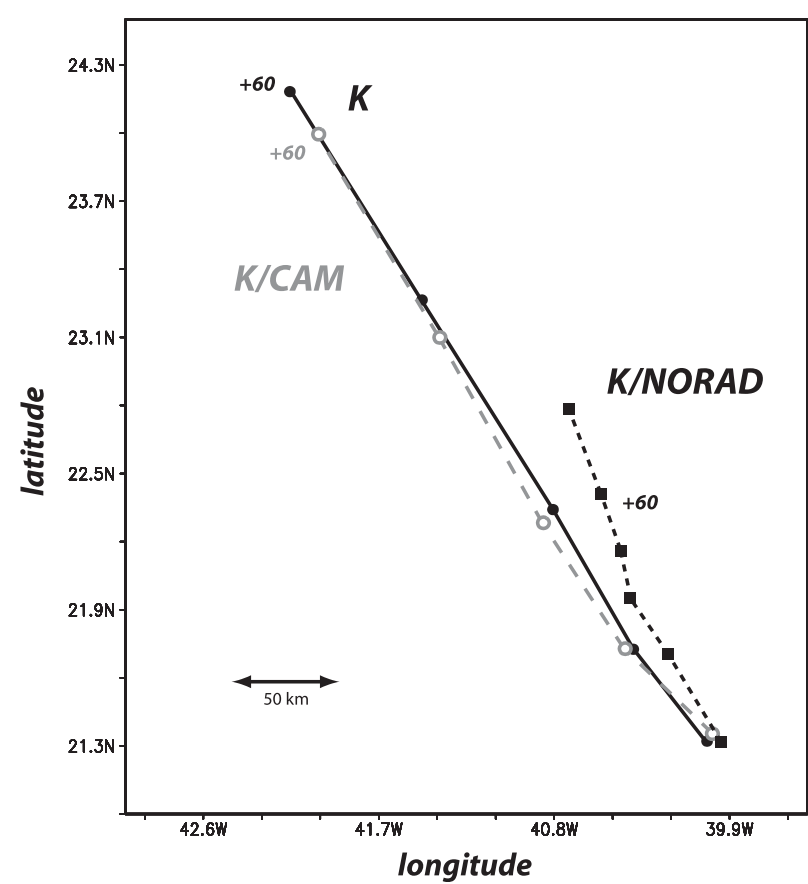

FIG. 9. As in Fig. 1, but comparing Kessler MP runs with the RRTM (K), CAM (K/CAM), and no radiation (K/NORAD) schemes.

on arguable and easily alterable assumptions involving particle geometry, density, and size distributions, as well as everything that controls how mass is transferred among condensate species. As dramatic examples of fall speed sensitivity, consider simulations with a version of Kessler in which all particles immediately became rain (K/NOCLOUD) and an LFO simulation in which graupel formation was prevented (L/NOGRAUPEL). These storms developed distinctly different motions

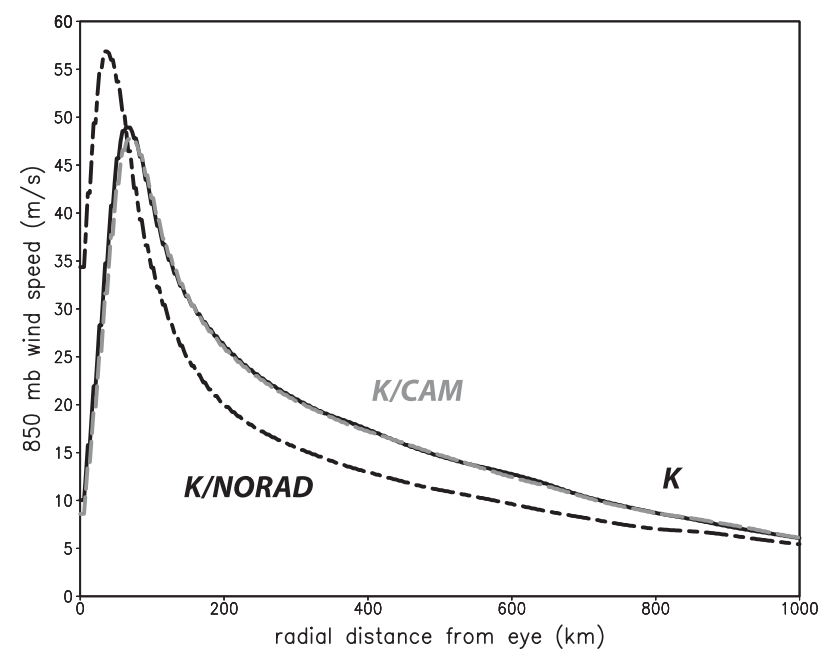

(Fig. 12), with the track in the K/NOCLOUD simulation resembling those of the original ice MP cases, whereas the L/NOGRAUPEL storm eventually became the most westward-propagating storm in this study.

With graupel production inhibited, the L/ NOGRAUPEL storm had an extremely extensive and thick anvil (Fig. 13) owing to the small but finite fall speed of snow particles. The virtual temperature anomaly distribution (Fig. 13) is likely due to the combination of subsidence (as in Fig. 7b) and a radiative effect that reflects the anvil's substantial depth. The winds at outer radius were relatively strong (Fig. 14) and exceeded those of the Kessler simulation beyond 500 $\mathrm{km}$. In contrast, the relative humidity cross sections in Fig. 15 demonstrate that the K/NOCLOUD storm that literally lacked cloud water had a markedly different anvil structure than the original Kessler storm (cf. Figs. 15a,b). Owing to considerably larger average particle fall speeds, the anvil extent in the K/NOCLOUD simulation is more comparable to those from the ice schemes (Figs. 7b,c).

The foregoing sensitivity tests suggest that manipulating microphysical properties influencing the storm anvil can lead to significant track deviations. However, other, considerably more subtle, ways of altering the radial pressure and temperature gradients via microphysical assumptions may also materially influence storm motion. Consider the track for the K/NOCLOUD2 simulation in Fig. 12a, which is a version of K/NOCLOUD in which all condensate became rain but the fall speed was set to zero in a truncated annular region that consisted of the lowest $5 \mathrm{~km}$ for columns with SLPs that exceeded $1005 \mathrm{mb}$. The inner radius of this annular zone was roughly $400 \mathrm{~km}$ beyond the storm center at all times (see areas delineated on Fig. 15c). This imposed a particle size

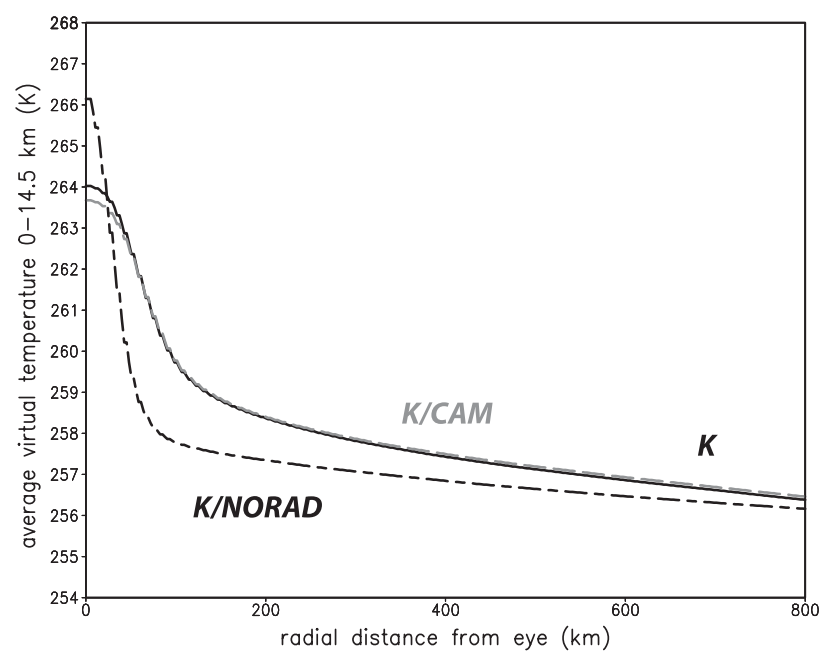

FIG. 10. As in Figs. 2 and 6, but comparing the K, K/CAM, and K/NORAD simulations. 


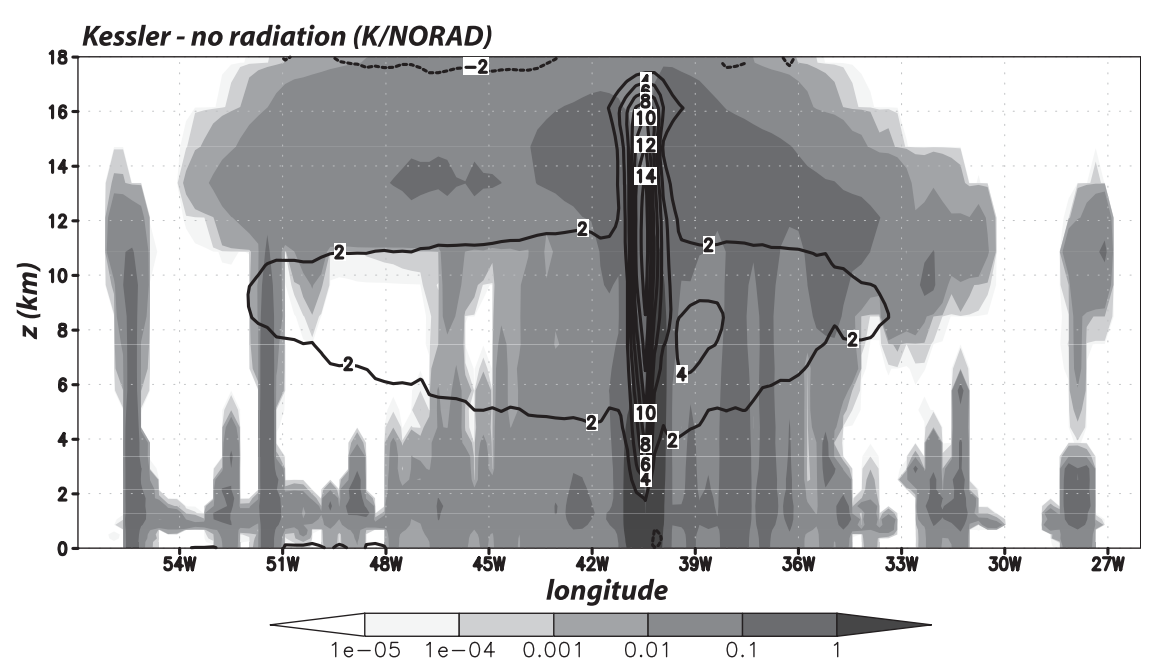

FIG. 11. As in Fig. 7a, except for the no-radiation Kessler simulation (K/NORAD).

and fall speed difference between the storm's inner and outer regions while leaving the storm anvil essentially unaltered. ${ }^{1}$ The purpose of this experiment is to demonstrate another manner in which radial temperature variations leading to storm motion differences could issue directly or indirectly from microphysical assumptions.

Because the K/NOCLOUD2 setup also produced large terminal velocities in the inner region where most of the condensation was generated, its anvil extent (Fig. 15c) was comparable to those of K/NOCLOUD and the ice MP storms. Comparison of Figs. 15b and 15c suggests that relative humidities differ little for the two no-cloud cases, at least above the lower troposphere. However, notice that the K/NOCLOUD2 storm moved in nearly the same direction as the original Kessler vortex (Fig. 12a). An explanation for the motion differences is sought in the radial profiles of $850-\mathrm{mb}$ wind speed and column average virtual temperature for these three versions of the Kessler MP (Fig. 16). The slower, more northerly motion in the K/NOCLOUD simulation is consistent with the weaker winds at outer radius, whereas the wind profile in the K/NOCLOUD2 case closely resembles that of the original Kessler run. Without anvil warming in the K/NOCLOUD2 simulation, the $0-14.5-\mathrm{km}$ layer $\bar{T}_{v}$ was systematically lower beyond the core, but it still had nearly the same radial gradient as in the $\mathrm{K}$ simulation. Based on the reasoning in section 3, it is reasonable that the Kessler and K/NOCLOUD2 vortices had comparable tracks, despite the absence of an extensive, radiatively important anvil in the latter.

\footnotetext{
${ }^{1}$ If precipitation particles were to enter the zero fall speed zone from above, they would cease falling (relative to still air), but the lack of an anvil above the zone minimizes this concern.
}

The relatively large radial temperature gradient in the K/NOCLOUD2 simulation (Fig. 16, right) arose because inclusion of the far-field zero fall speed zone resulted in higher (lower) temperatures near (far from) the core relative to the K/NOCLOUD case. Differences are also apparent in the condensate and virtual temperature perturbation fields (Fig. 17). In both simulations, cumulus clouds were periodically generated in the outer rainband zone at large distances from the core. The net effect these clouds have on the main storm inflow region is a complex combination of condensation, evaporation, and fallout rates, along with fluxes from the sea surface. In the K/NOCLOUD2 case, particles created in the zero fall speed annulus were precluded from falling, which led to longer residence times and a greater likelihood of evaporating. This evaporation undoubtedly contributed to the cooling (Fig. 17b versus Fig. 17a) and moistening (Fig. 15c versus Fig. 15b) of the air in the annulus relative to the same space in the other no-cloud simulation.

Moistening of the far-field environment in the K/NOCLOUD2 case probably led to the higher temperatures closer to the core through enhanced inward moisture advection by the low-level radial inflow. Comparing the column total condensate for these two nocloud simulations (Fig. 18), a clear difference in convective activity is seen that is not restricted to the no-fallout annulus (i.e., the region beyond the black circle in Fig. 18b). The combination of remote cooling and inner radius warming in the K/NOCLOUD2 storm increased the radial temperature and pressure gradients and led to more intense winds at larger radii, which then contributed to the track differences between the nocloud simulations in Fig. 12a. Although somewhat unphysical, the K/NOCLOUD2 experiment suggests a 

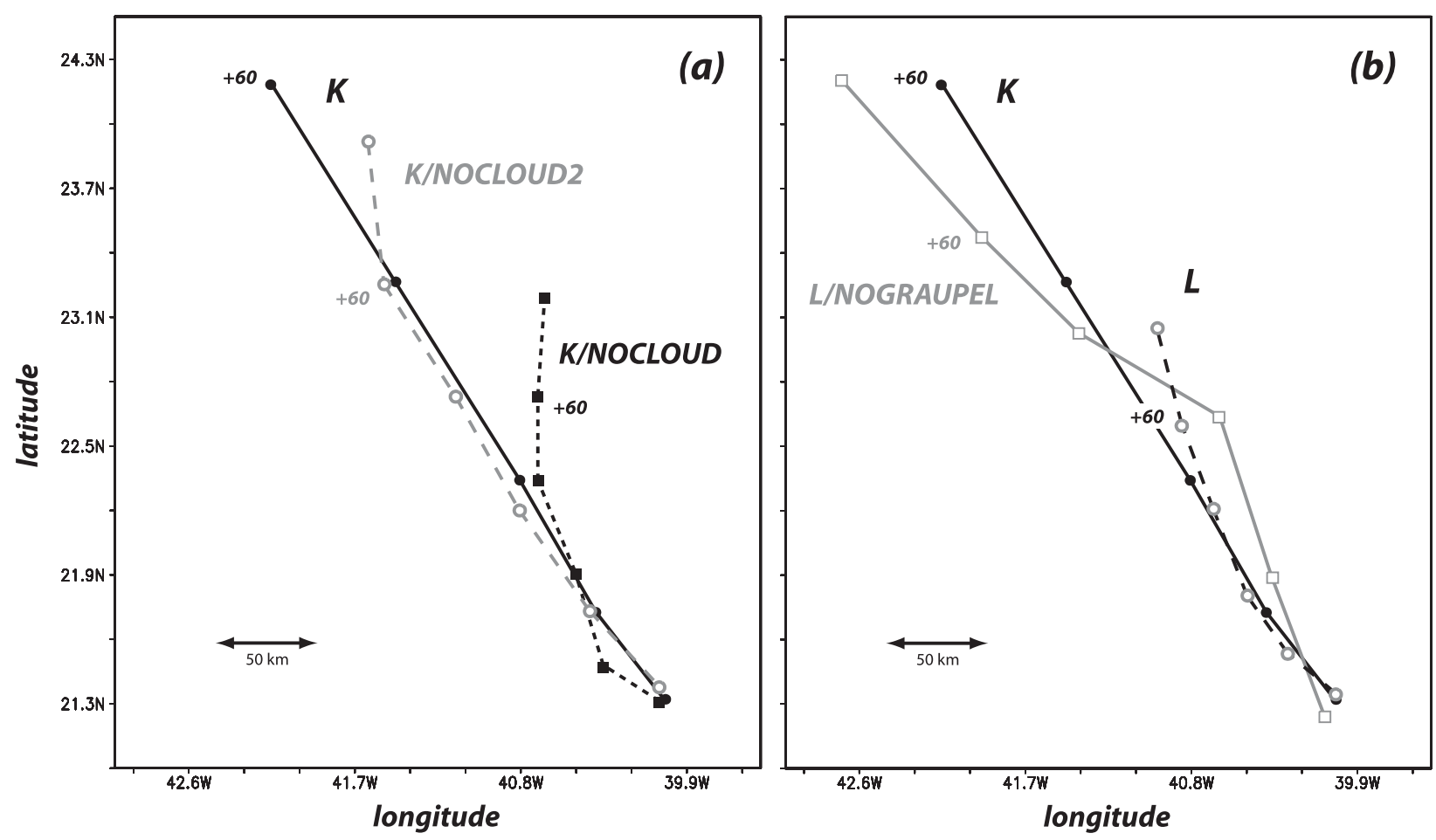

FIG. 12. As in Fig. 1, but contrasting the storm motion in the original Kessler simulation (K) with (a) two no-cloud versions of the Kessler scheme (K/NOCLOUD and K/NOCLOUD2) and (b) a modified LFO run without graupel (L/NOGRAUPEL). See text for descriptions of these sensitivity tests.

means by which shallower convection at significant distance from the cyclone core can exert an important influence on the storm motion.

As acknowledged earlier, other factors besides those captured in FE89's model may influence storm motion and flow across the vortex. These other influences must be used to explain the $4 \mathrm{~km} \mathrm{~h}^{-1}$ propagation difference between the K/NOCLOUD2 and the original Kessler vortices (Fig. 12a). Whereas the lower tropospheric winds were comparable (Fig. 16), the speed and direction differences were found to be consistent with the 900-200-mb layer-mean wind averaged over radii of 400 and $200 \mathrm{~km}$ for the $\mathrm{K}$ and K/NOCLOUD2 storms, respectively. Similar layer depths have been found to be

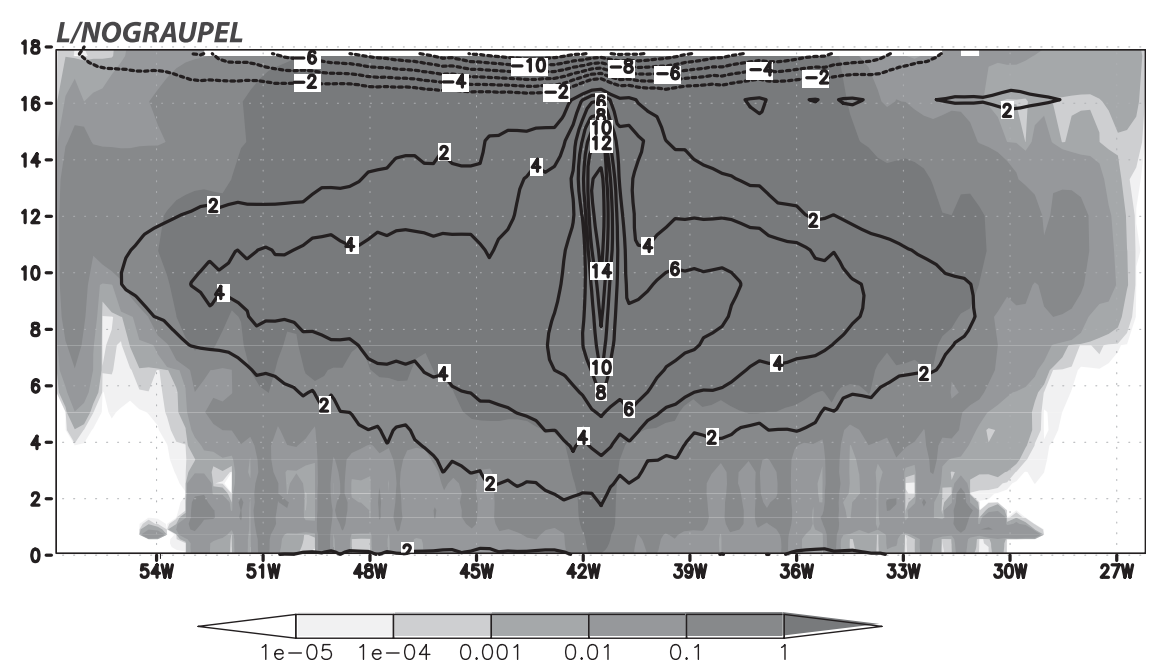

FIG. 13. As in Fig. 7b, but for the L/NOGRAUPEL run. 


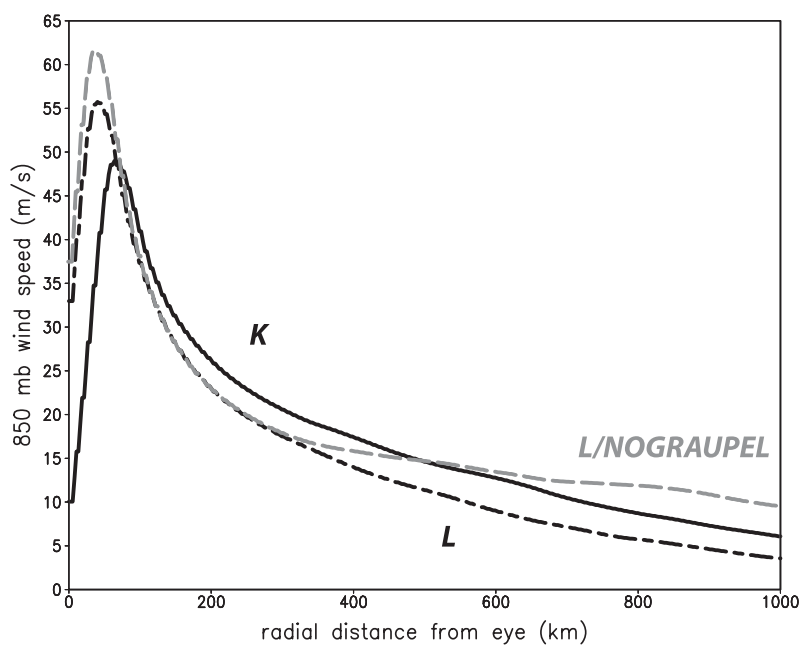

FIG. 14. As in Fig. 2, but comparing the K, L, and L/NOGRAUPEL cases.

skillful in other studies (e.g., Chan and Gray 1982; Ritchie and Frank 2007). It is not immediately clear why the appropriate averaging radii are different for the two cases.

It is noted in passing that both Kessler no-cloud simulations possessed narrower eyes with maximum near-surface winds about $20 \%$ stronger than the original Kessler run (Fig. 16), with both exceeding that of the standard LFO run (Fig. 2). The influence of cloud microphysical assumptions on storm intensity has been appreciated for some time (e.g., Lord et al. 1984; McFarquhar et al. 2006; Zhu and Zhang 2006). These results suggest that average particle fall speeds are somehow playing an indirect or direct role.

\section{Discussion and summary}

FS07 demonstrated that varying cloud microphysics and cumulus schemes in a single model could produce hurricane motion deviations resulting in significantly different track forecasts, including track spreads comparable to multimodel ensembles, even in relatively short ( 2 day) simulations. This work extends that study by examining how and why cloud microphysical assumptions can directly or indirectly influence storm motion. Idealized simulations were made in a dynamical framework that included model physical processes (such as boundary layer, surface, and radiation schemes) that are typically employed in operational forecasting models. The exclusion of land and initial environmental variations facilitated interpretation of these simulations.

This work presumes that vortex motion in an environment with no initial basic current is substantially (though not solely) dependent on the strength of the symmetric flow far beyond the storm core. FE89 demonstrated that the tangential winds at the outer radius ( $>300 \mathrm{~km}$ from the core) determined the speed and also direction of their cyclonic vortices in environments with no basic current because those winds determined the strength and orientation of beta gyres responsible for storm motion in their simple model. To a high degree of accuracy, these winds are in gradient balance with respect to the radial pressure distribution, which is a reflection of the gradient of deep-layer mean virtual temperature $\bar{T}_{v}$.

It is this radial temperature structure that the cloud microphysics representation in the model can modulate in a variety of ways. For example, it was shown that microphysics schemes (such as Kessler) resulting in a smaller average particle fall speed lead to anvil enhancement that (other factors being equal) can influence the track via cloud-radiative interaction. Such an anvil induces local warming below, and cooling above, the anvil cloud layer. Integrating downward from the upper tropospheric level where radial pressure gradients vanish revealed that extensive anvils not only raised the layer-mean temperature but also, more importantly, increased its radial gradient and thereby changed the radial gradient of surface pressure and ultimately the track. A similar result was obtained when the balance between slowly falling snow and more quickly falling graupel particles was altered in the LFO scheme.

Because the warm rain parameterization appears to encourage excessive anvil cloud formation, it seems prudent to remove or restrain cloud-radiative feedbacks when that MP is used. However, one should not focus on a single scheme (Kessler) or storm characteristic (the anvil cloud), especially because the no-cloud sensitivity tests (K/NOCLOUD and K/NOCLOUD2) revealed that enhanced evaporative cooling in the lower troposphere at large radii could also substantially alter the radial $\bar{T}_{v}$ gradient and thus the track. One way of encouraging more evaporation anywhere is to decrease the mean particle size, which is tantamount to reducing the fall speed. The authors believe these results point to a sensitivity to fall speed partitioning between slower and faster falling condensate particles that may be more readily revealed in a simple scheme (like Kessler) but could also easily occur in more complex schemes depending on how interactions among condensate classes such as cloud ice, snow, and graupel are handled. In particular, schemes lacking the means of producing heavier, faster-falling particles in sufficient quantity may produce less realistic results.

Two limitations of this study must be noted. These simulations have relatively coarse model resolution at large radius because of the telescoping domains, which 
(a) Kessler (K)
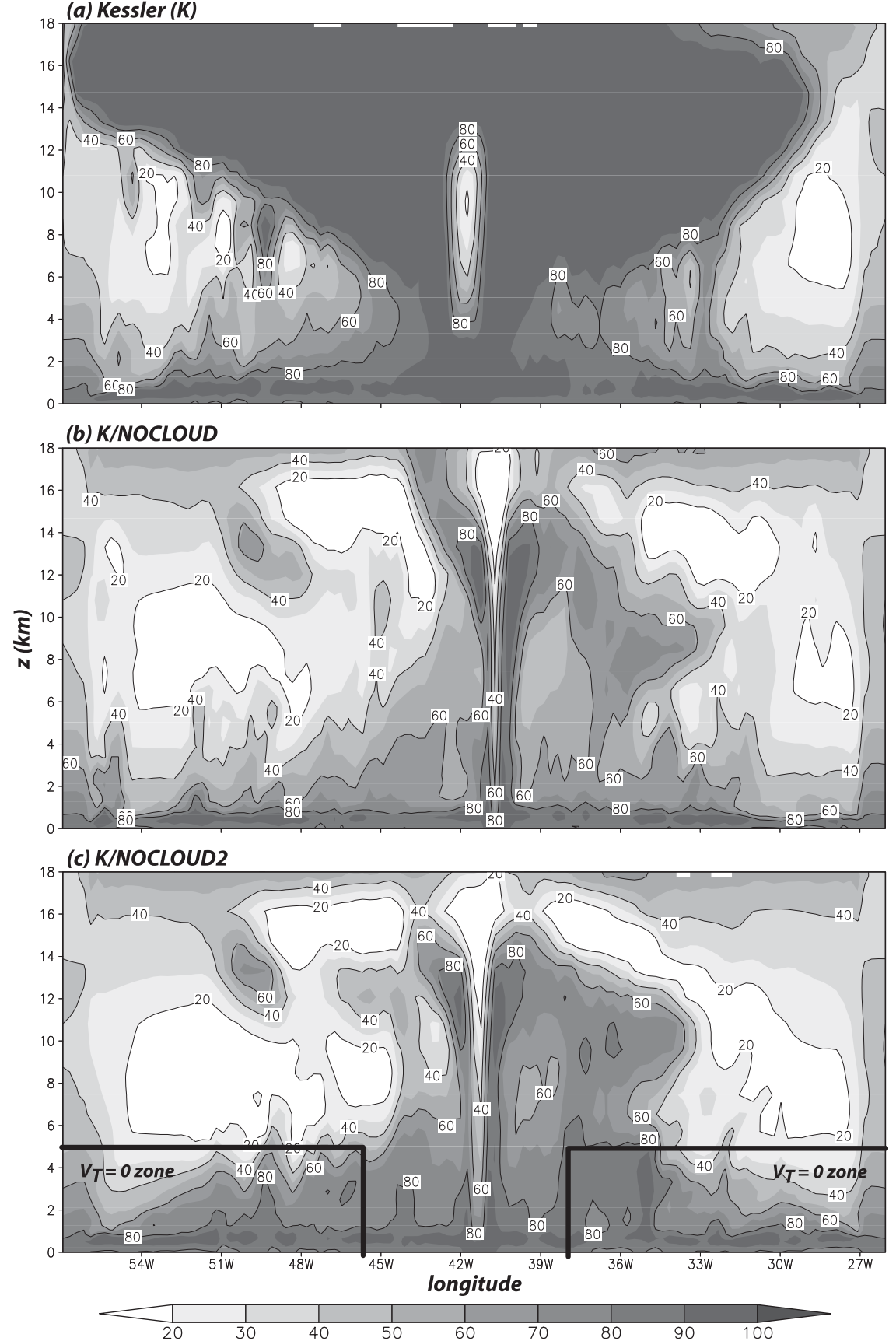

FIG. 15. Vertical cross sections of relative humidity for the (a) Kessler, (b) K/NOCLOUD, and (c) K/NOCLOUD2 storms. In (c), the areas of intersection of the zero fallout annulus with the vertical plane are indicated.

could affect the production of convective motions in outer regions and thus the across-storm gradients and track. However, preliminary work using uniform higher resolution at large radii has not revealed material changes in either the results or conclusions presented herein. Potentially more serious is the use of microphysical schemes containing parameters that were tuned for other applications and are thus likely to be less representative of tropical cyclones. For example, McFarquhar and Black (2004) documented some significant inconsistencies with respect to size distributions of frozen water between tropical cyclones and other kinds of convection. Additional comprehensive observations and more sophisticated microphysical schemes 

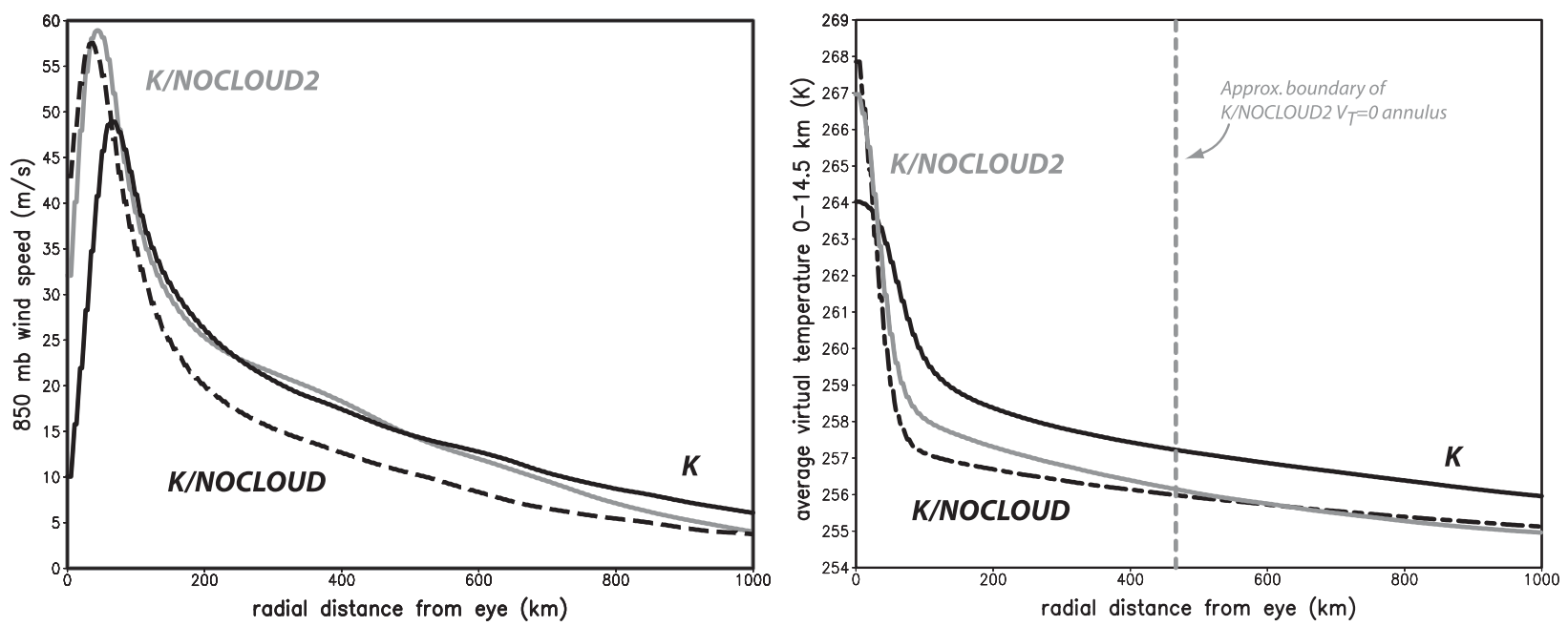

FIG. 16. As in Figs. 2 and 6, but for the Kessler, K/NOCLOUD, and K/NOCLOUD2 storms. On the panel at right, the approximate location of the zero fall speed annulus' boundary is shown.

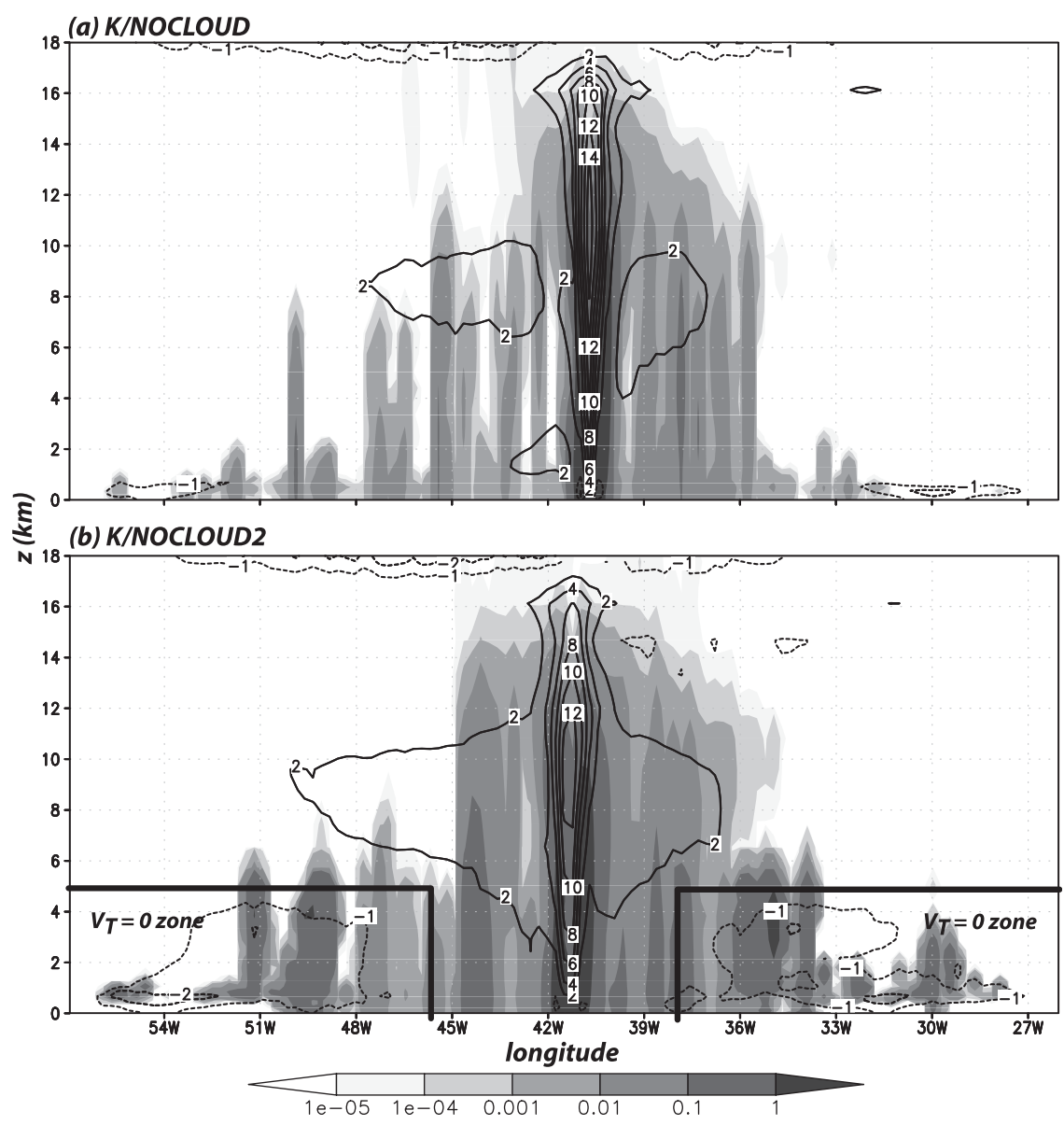

FIG. 17. As in Fig. 7, but for the K/NOCLOUD and K/NOCLOUD2 storms. Compare both to Fig. $7 \mathrm{a}$ 


\section{(a) K/NOCLOUD}

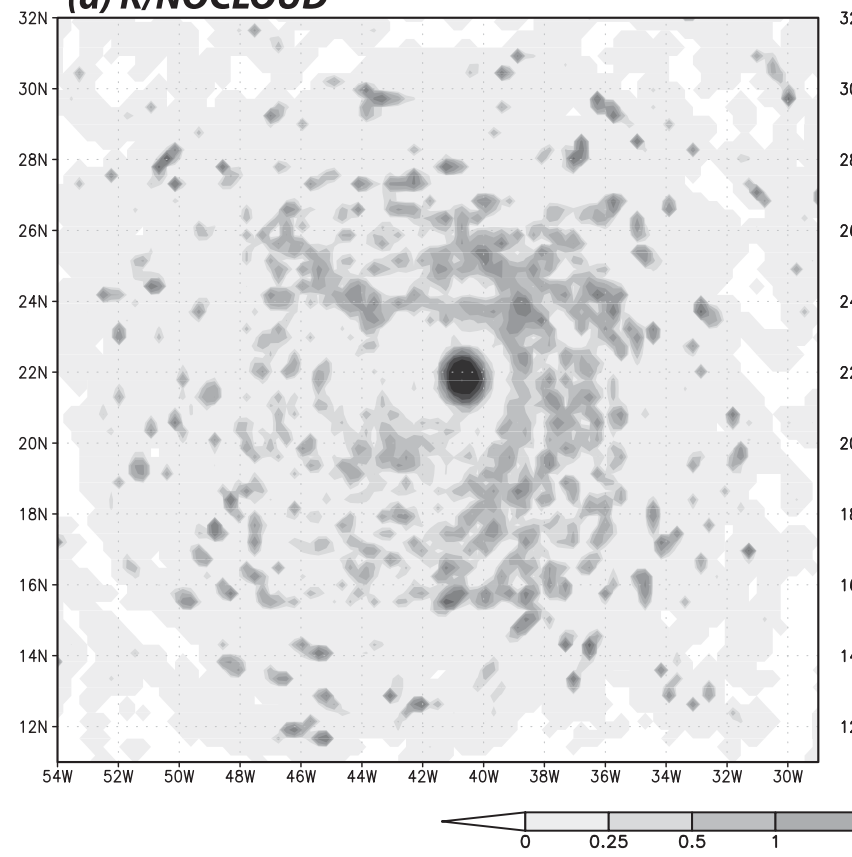

(b) K/NOCLOUD2

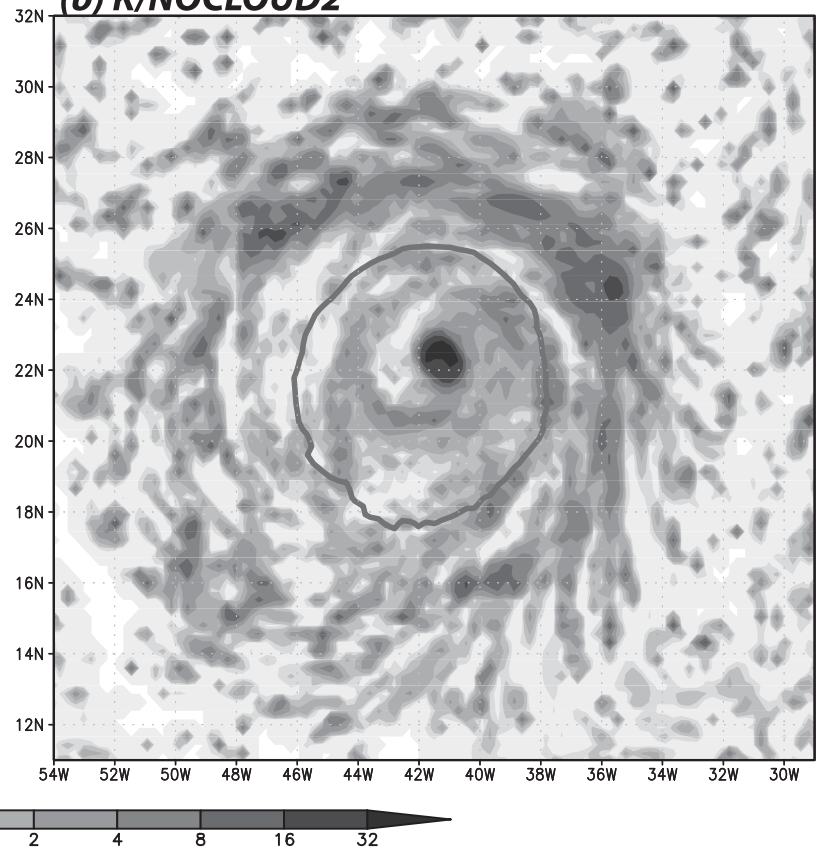

FIG. 18. Vertically integrated total condensate $\left(\mathrm{g} \mathrm{kg}^{-1}\right)$ averaged over a 12-h period for the (a) K/NOCLOUD and (b) K/NOCLOUD2 storms. In (b), the black circle indicates the average location of the 1005-mb SLP contour, which represents the inner boundary of the zero fall speed annulus.

are clearly needed. In fact, the difference between the two no-cloud simulations suggests that how outer rainbands are handled in operational models may potentially influence how the model storms move, thereby affecting position forecasts.

It should be kept in mind that other avenues of tapping into microphysical sensitivity besides via fall speeds and cloud-radiative interactions likely exist. The important point is that microphysical assumptions modulate temperature gradients that largely determine pressure gradients that generate the winds that help determine storm track. As FS07 hypothesized, differences in track forecasts arising from various dynamical models could partly reflect variations in microphysical representations in the models. Finally, note that microphysically induced differences in storm speed and direction in these idealized simulations could, in practice, become greatly amplified by spatial variations in atmospheric and surface conditions, including steering flows, vertical shears, dry and moist tongues, sea surface temperatures, and the relative arrangement of land and sea. These would lead to even larger ensemble track spreads.

Acknowledgments. Comments kindly provided by Dr. Russ Elsberry greatly improved the manuscript. The single-sounding WRF initialization was designed by Gary Lackmann and Kevin Hill (North Carolina
State University). RGF was supported by NSF Grant ATM-0554765. HCK was supported by Grants CQSE 97R0066-69 and NSC 97-2628-M-002-023. The support of The Aerospace Corporation is also acknowledged.

\section{REFERENCES}

Betts, A. K., and M. Miller, 1986: A new convective adjustment scheme. Part II: Single column tests using GATE wave, BOMEX, ATEX and arctic air-mass data sets. Quart. J. Roy. Meteor. Soc., 112, 693-709.

Black, M. L., R. W. Burpee, and F. D. Marks, 1996: Vertical motion characteristics of tropical cyclones determined with airborne Doppler radial velocities. J. Atmos. Sci., 53, 1887-1909.

Chan, J. C. L., and W. M. Gray, 1982: Tropical cyclone movement and surrounding flow relationships. Mon. Wea. Rev., 110, $1354-1374$.

- and R. T. Williams, 1987: Analytical and numerical studies of the beta-effect in tropical cyclone motion. Part I: Zero mean flow. J. Atmos. Sci., 44, 1257-1265.

Fiorino, M. J., and R. L. Elsberry, 1989: Some aspects of vortex structure related to tropical cyclone motion. J. Atmos. Sci., 46, 975-990.

Flatau, M., W. H. Schubert, and D. E. Stevens, 1994: The role of baroclinic processes in tropical cyclone motion: The influence of vertical tilt. J. Atmos. Sci., 51, 2589-2601.

Fovell, R. G., and Y. Ogura, 1988: Numerical simulation of a midlatitude squall line in two dimensions. J. Atmos. Sci., 45, 3846-3879. 
and H. Su, 2007: Impact of cloud microphysics on hurricane track forecasts. Geophys. Res. Lett., 34, L24810, doi:10.1029/ 2007GL031723.

—, G. L. Mullendore, and S.-H. Kim, 2006: Discrete propagation in numerically simulated nocturnal squall lines. Mon. Wea. Rev., 134, 3735-3752.

Hawkins, H. F., and D. T. Rubsam, 1968: Hurricane Hilda, 1964. II: Structure and budgets of the hurricane on October 1, 1964. Mon. Wea. Rev., 96, 617-636.

Hill, K. A., and G. M. Lackmann, 2009: Analysis of idealized tropical cyclone simulations using the Weather Research and Forecasting model: Sensitivity to turbulence parameterization and grid spacing. Mon. Wea. Rev., 137, 745-765.

Holland, G. J., 1983: Tropical cyclone motion: Environmental interaction plus a beta effect. J. Atmos. Sci., 40, 328-342.

Jordan, C. L., 1958: Mean soundings for the West Indies area. J. Meteor., 15, 91-97.

Kain, J. S., and J. M. Fritsch, 1993: Convective parameterization for mesoscale models: The Kain-Fritsch scheme. The Representation of Cumulus Convection in Numerical Models, Meteor. Monogr., No. 24, Amer. Meteor. Soc., 165-170.

Kidder, S. Q., M. D. Goldberg, R. M. Zehr, M. DeMaria, J. F. W. Purdom, C. S. Velden, N. C. Grody, and S. J. Kusselson, 2000: Satellite analysis of tropical cyclones using the Advanced Microwave Sounding Unit (AMSU). Bull. Amer. Meteor. Soc., 81, 1241-1259.

Knaff, J. A., S. A. Seseske, M. DeMaria, and J. L. Demuth, 2004: On the influences of vertical wind shear on symmetric tropical cyclone structure derived from AMSU. Mon. Wea. Rev., 132, 2503-2510.

Kossin, J. P., 2002: Daily hurricane variability inferred from GOES infrared imagery. Mon. Wea. Rev., 130, 2260-2270.

Lord, S. J., H. E. Willoughby, and J. M. Piotrowicz, 1984: Role of a parameterized ice-phase microphysics in an axisymmetric, nonhydrostatic tropical cyclone model. J. Atmos. Sci., 41, 2836-2848.

Markowski, P. M., E. N. Rasmussen, J. M. Straka, and D. C. Dowell, 1998: Observations of low-level baroclinicity generated by anvil shadows. Mon. Wea. Rev., 126, 2942-2958.
Marks, F. D., P. G. Black, M. T. Montgomery, and R. W. Burpee, 2008: Structure of the eye and eyewall of Hurricane Hugo (1989). Mon. Wea. Rev., 136, 1237-1259.

McFarquhar, G. M., and R. A. Black, 2004: Observations of particle size and phase in tropical cyclones: Implications for mesoscale modeling of microphysical processes. J. Atmos. Sci., 61, 422-439.

_ - H. Zhang, G. Heymsfield, R. Hood, J. Dudhia, J. B. Halverson, and F. Marks, 2006: Factors affecting the evolution of Hurricane Erin (2001) and the distributions of hydrometeors: Role of microphysical processes. J. Atmos. Sci., 63, 127-150.

Mlawer, E. J., S. J. Taubman, P. D. Brown, M. J. Iacono, and S. A Clough, 1997: Radiative transfer for inhomogeneous atmospheres: RRTM, a validated correlated- $k$ model for the longwave. J. Geophys. Res., 102 (D14), 16 663-16 682.

Nolan, D. S., M. T. Montgomery, and L. D. Grasso, 2001: The wavenumber-one instability and trochoidal motion of hurricane-like vortices. J. Atmos. Sci., 58, 3243-3270.

Ritchie, E. A., and W. M. Frank, 2007: Interactions between simulated tropical cyclones and an environment with a variable Coriolis parameter. Mon. Wea. Rev., 135, 1889-1905.

Stensrud, D. J., 2007: Parameterization Schemes: Keys to Understanding Numerical Weather Prediction Models. Cambridge University Press, 488 pp.

Wang, Y., 2002: An explicit simulation of tropical cyclones with a triply nested movable mesh primitive equation model: TCM3 Part II: Model refinements and sensitivity to cloud microphysics parameterization. Mon. Wea. Rev., 130, 3022-3036.

_ Part II: Diabatic vortices. J. Atmos. Sci., 53, 3727-3756.

Wu, C.-C., and K. A. Emanuel, 1993: Interaction of a baroclinic vortex with background shear: Application to hurricane movement. J. Atmos. Sci., 50, 62-76.

Zhang, D.-L., E.-Y. Hsie, and M. W. Moncrieff, 1988: A comparison of explicit and implicit predictions of convective and stratiform precipitating weather systems with a meso- $\beta$-scale numerical model. Quart. J. Roy. Meteor. Soc., 114, 31-60.

Zhu, T., and D.-L. Zhang, 2006: Numerical simulation of Hurricane Bonnie (1998). Part II: Sensitivity to varying cloud microphysical processes. J. Atmos. Sci., 63, 109-126. 